\title{
Der Pflegedienst im Fokus - Aus- und Weiterbildung bei HELIOS: der Kompetenzkompass
}

\author{
Mandy Dietel
}

(C) Springer-Verlag 2012

\section{Hintergrund}

In den HELIOS Kliniken wurde auf Initiative der Fachgruppe Pflege nach einem neuen Instrument gesucht, mit dem die Kompetenzen der Auszubildenden in allen drei Ausbildungsjahren erfasst werden können. Mit der Frage „Was erwarten wir von unseren zukünftigen Kolleginnen im Pflegebereich?" begann die AG Kompetenzen im November 2009 mit ihren Entwicklungen.

\section{Methode}

Die Grundlage für den Kompetenzkompass bilden einerseits die zwölf Themenbereiche der theoretischen Ausbildung nach der Ausbildungs- und Prüfungsverordnung und andererseits die vier Schlüsselkompetenzen 1t. Krankenpflegegesetz. Die zentrale Fragestellung bezieht sich darauf, wie stark die Kompetenzen innerhalb der einzelnen Themenbereiche ausgeprägt sind. Neben der Beschreibung der einzelnen Themenbereiche, werden bei der Kompetenzmessung inhaltliche Schwerpunkte gesetzt. Um dies in der Praxis für die Auszubildenden und die Praxisanleiterinnen zu veranschaulichen, wurde eine Grafik zur Kompetenzeinschätzung entwickelt. Auf diesem sogenannten Kompetenzstrahl erfolgt die Kompetenzmessung regelhaft und unter Berücksichtigung des Ausbildungsstandes. Die Visualisierung der Kompetenzentwicklung übernehmen die Auszubildenden selbst, indem jede Einschätzung zu den Themenbereichen in eine Grafik und in eine Tabelle eingetragen wird. Die Eigenverantwortlichkeit der Auszubildenden steht dabei im Mittelpunkt. In einem Einführungs-, Zwischen- und

\begin{abstract}
Abschlussgespräch werden die Lernziele für die Auszubildenden gemeinsam mit den Praxisanleiterinnen festgelegt, überprüft sowie reflektiert und innerhalb der Gesprächsprotokolle dokumentiert. Um gezielt bestimmte Techniken einzuüben ist weiterhin ein Protokoll zu den sogenannten Skills (klinische Fertigkeiten) entstanden. Die Auszubildenden haben somit die Möglichkeit, unter Anleitung die Techniken zu wiederholen. Um eine erfolgreiche Implementierung gewährleisten zu können, werden alle Praxisanleiterinnen und Auszubildenden im Umgang mit dem Kompetenzkompass geschult.
\end{abstract}

\section{Ergebnisse und Diskussion}

Der Kompetenzkompass ist ein Instrument, in dem das komplexe berufliche Handeln in der Pflege in den fachlichen, personalen, sozialen und methodischen Kompetenzen sichtbar wird. Dies löst eine vorrangig tätigkeitsorientierte Ausbildung ab. Die kontinuierliche Begleitung und transparente Dokumentation ermöglicht weiterhin eine objektive Bewertung und sichert mittels strukturierter Lernziele eine kontinuierlich hohe Qualität. Der Kompetenzkompass fördert die Eigenverantwortlichkeit der Auszubildenden und stellt zusammenfassend die professionelle Entwicklung in den drei Ausbildungsjahren dar. Erste Ergebnisse des Pilotprojektes, welches im April diesen Jahres in der HELIOS Klinik Titisee-Neustadt gestartet ist, erwarten wir im November 2011. Nach Implementierung in allen HELIOS Kliniken erfolgt eine umfassende Evaluation des Kompetenzkompass. 